\title{
Sensing Uncertainty Reduction Using Low Complexity Actuation
}

\author{
Aman Kansal, Eric Yuen, William J Kaiser, Gregory J Pottie and Mani B Srivastava \\ Department of Electrical Engineering \\ University of California, Los Angeles \\ Los Angeles, USA \\ \{kansal, eyuen, kaiser, pottie, mbs\}@ee.ucla.edu
}

\begin{abstract}
The performance of a sensor network may be best judged by the quality of application specific information return. The actual sensing performance of a deployed sensor network depends on several factors which cannot be accounted at design time, such as environmental obstacles to sensing. We propose the use of mobility to overcome the effect of unpredictable environmental influence and to adapt to run time dynamics. Now, mobility with its dependencies such as precise localization and navigation is expensive in terms of hardware resources and energy constraints, and may not be feasible in compact, densely deployed and widespread sensor nodes. We present a method based on low complexity and low energy actuation primitives which are feasible for implementation in sensor networks. We prove how these primitives improve the detection capabilities with theoretical analysis, extensive simulations and real world experiments. The significant coverage advantage recurrent in our investigation justifies our own and other parallel ongoing work in the implementation and refinement of self-actuated systems.
\end{abstract}

\section{Categories and Subject Descriptors}

B.8 [Hardware]: Performance and Reliability; C.2.4 [Computer Communication Networks]: Distributed Systems—distributed applications

\section{General Terms}

Algorithms, Measurement, Reliability, Experimentation

\section{Keywords}

sensor networks, coverage, actuation, environmental obstacles

\section{INTRODUCTION}

Several research efforts $[1,2,3,4,5,6,7,8]$ have established the feasibility of compact, wireless and low energy devices for sensor networks. Apart from use in defense and industrial process monitoring, several other applications have been prototyped for such

Permission to make digital or hard copies of all or part of this work for personal or classroom use is granted without fee provided that copies are not made or distributed for profit or commercial advantage and that copies bear this notice and the full citation on the first page. To copy otherwise, to republish, to post on servers or to redistribute to lists, requires prior specific permission and/or a fee.

IPSN'04, April 26-27, 2004, Berkeley, California, USA

Copyright 2004 ACM 1-58113-846-6/04/0004 ...\$5.00. systems in education $[7,6]$, science $[9,10]$, arts and entertainment [11] among other areas. In this work we consider the fundamental functionality used by all the above applications - sensing the application specific phenomenon in the deployment environment. Any given application would need a certain sensing performance, which must be guaranteed in the face of unpredictable event distributions and the presence of static and mobile environmental obstacles. Such practical considerations are critical in the design of sensor networks due to the strong coupling of the system to its deployment environs. For instance, if a network of cameras is installed for security monitoring and certain regions of the scene are occluded by obstacles, the utility of the system to the user will immediately diminish. The system designer is challenged to not only provide the required performance within the resource constraints of embedded sensor nodes and a limited power budget but also ensure autonomous operation of the system in unknown environments. Environment specific customization is not desirable, as it hinders rapid deployment.

\subsection{Key Contributions}

The performance problem outlined above can be alleviated with the use of mobility. We argue that the use of mobility to overcome sensing uncertainties is essential due to two reasons.

The first reason is providing sensor diversity. A certain node density may be calculated for a specific sensor based on its nominal range in an isotropic medium or the required granularity at which the phenomenon is to be sensed. However, this density does not guarantee the quality of sensing in a real environment with anisotropic media and presence of obstacles. A prohibitively high density would be required to guarantee performance in an unknown and arbitrary environment. Thus, while static high density sensors can give only probabilistic coverage performance, actuated sensors can adapt to the specific deployment scenario and give the exact coverage performance required.

The second reason comes from the need for adapting to the run time dynamics of the environment. Obstacles may move in an unpredictable manner making it necessary for the system to adjust. Thus, the only feasible method to achieve performance guarantees is to endow the system with a capability to auto-configure and reposition in response to environmental peculiarities.

We show in this paper that a small range of mobility relative to the mean obstacle size of the environment can lead to significant improvement in coverage performance. This finding is extremely important for sensor network design because of the specific nature of resource constraints in the system. Mobility in a general form has several disadvantages which make its use in sensor networks highly impractical. Most proposed systems which do use mobil- 
ity restrict it to a small subset of the nodes in the network [12, 13]. Firstly, supporting mobility requires the nodes to be capable of accurately localizing their positions and navigating across the deployed terrain. This requires significant resources in terms of localization hardware, terrain sensing, motion feedback and the resultant complex data processing. Secondly, even if all the resources can be provided, the errors in the position of the node itself introduce further complications in the sensing and detection algorithms. Thirdly, large amounts of energy are required for physically moving the node on arbitrary terrain.

In contrast, node mobility operating over a limited range can feasibly be provided to low cost sensor nodes deployed in large numbers. Several factors lead to this conclusion. First, a short linear displacement may be executed through addition of small infrastructure in the form of linear actuators, for example. This may include a track mechanism, permitting motion as indicated in (Figure 1). Adding locomotion to compact nodes is feasible, as demonstrated on Robomotes [14], also shown in Figure 1.

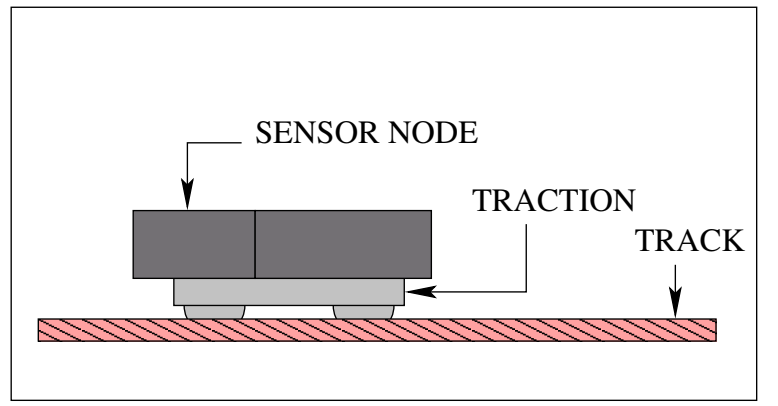

(a)

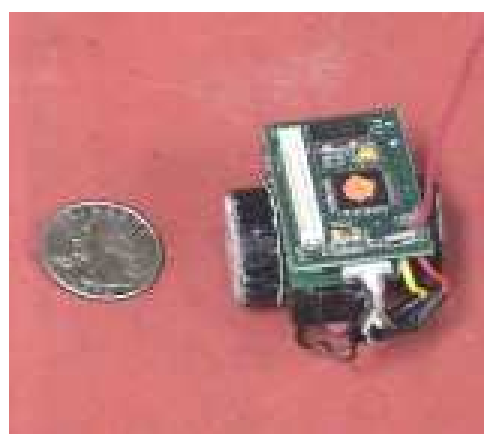

(b)

Figure 1: (a) Conceptual visualization of feasible motion support in sensor nodes, (b) Robomote: a sensor node with a small traction mechanism [14]

In addition, energy requirements for moving on a pre-designed infrastructure are low since the node is not subjected to arbitrary terrain navigation characteristics. Further, when the mobility is limited to a pre-designed platform, counterbalancing weights may be introduced to reduce the energy cost of motion due to gravitational forces. Moreover, for this application, only the sensor transducer is required to be moved instead of the entire node. Accurate localization, real time terrain feedback and complex navigation is no longer required for the purposes of mobility as the track is deterministic (the application specific localization, if used remains independent). Relative positions along the designed locomotion track are conveniently recorded and this motion does not add to detection uncertainty. Further, limited range mobility can be much faster than unconstrained mobility due to its simplified navigation requirements and hence reduced latency. Thus, our work presents a practical low complexity and low resource cost method for overcoming the effect of environmental obstacles and run time dynamics.

\subsection{Related work}

Most works on coverage and sensing performance in sensor networks assume simple sensing range models and ignore the effect of obstacles $[15,16]$. Somewhat more involved sensing models were developed in [17] but obstacles were not accounted for. Some work has also been done on saving energy by turning off redundant nodes while maintaining coverage and connectivity but the proposed algorithms assume that coverage is not blocked due to environmental occlusions [18, 15, 19, 20]. However, most environments do pose obstructions in the paths of sensors and performance may be affected severely by these. Our work addresses the modelling of realistic environmental obstacles in sensor networks for the first time.

The problem of communication coverage in various environments with obstacles has been widely studied in cellular communication and more recently in sensor networks [21] but such analysis is not applicable to sensing coverage. This is because the propagation of phenomenon information through sensing media is very different from radio wave propagation. Techniques to model path loss, reflections and diffractions for electromagnetic waves, their lognormal approximations and empirically derived models [22] are not applicable for most sensors. In particular, the image sensor studied in this work depends on direct line of sight access to the phenomenon of interest, which is not the case for cellular communication.

The problem of observing and reconstructing a given scene from images acquired by single or multiple cameras has been studied extensively in vision research. Primarily, research has concentrated on complete descriptions of the observable scene [23], sampling the scene with the minimum required views to achieve a complete description [24], planning the placement of cameras [25] and moving the camera in response to detected obstacles [26]. The vision systems assumed are relatively free to move as required or can be deployed at the optimal positions computed for a specific environment, which is not the case for sensor networks. In addition, the processing and energy resource constraints are very different in these systems compared to sensor networks. We concentrate on systems where only a limited amount of mobility is available as discussed above. Previous work has also considered path planning for robots in the presence of local obstacles and tracking of moving targets in the presence of occlusions [27, 28, 29]. Again, the robots in such systems have extensive navigational and localization resources and are capable of moving much more freely than is practical for sensor nodes.

The use of mobility to improve the performance of ad hoc wireless networks has been considered before to enhance communication capacity [30] and security [31]. Some implementations to exploit externally available motion to transfer data physically instead of over the wireless links [13, 32, 33, 34] have been considered. The problem of target detection by randomly moving sensor nodes has also been looked at [35]. All of these works assume that the mobility is random or available by some other means such a predictable path of a vehicle passing through the deployment. They do not consider actuation built into the sensor nodes.

Other uses of mobility such as self-deployment [36] and energy 
replenishment [12] have been studied, but these methods are not based on low complexity actuation. The only other work, which we are aware of, to utilize controlled mobility for enhancing sensor coverage is [37]. However, that work aims at maintaining a uniform node density in the event of node deaths and does not address the issue of obstacles and sensing medium anisotropies.

\subsection{Outline}

In the next section, we theoretically analyze the effect of an obstacle, and how a small range of mobility affects the sensing performance. In section 3 we consider the advantages of mobility for several cases conceived to model complex environments with multiple sensor deployment possibilities. Section 4 presents the observations from our experiments conducted with real sensors, both on a laboratory test-bed and in a real world deployment. We assess the observations from simulations and experiments in section 5 and conclude the paper in section 6 .

\section{ANALYZING THE EFFECT OF ENVIRONMENTAL OBSTACLES}

We first analyze the effect of an obstacle on coverage. For the purpose of analysis we make several abstractions which will be removed gradually in simulations, laboratory tests and real world experiments presented in subsequent sections. We carry out our analysis for a sensor with line of sight coverage model, such as a camera, for ease of exposition; the analysis can be extended to sensors in anisotropic media, acoustic sensors in a multi-path environment and other sensor specific coverage models.

Assume that sensors are deployed at uniform density $d$ which yields an inter-sensor spacing $a$ in a regular grid deployment. Most of the deployments stay close to the ground surface and for such cases a two dimensional deployment is a reasonable model. Consider one sensor which is responsible for covering one tile of this grid, shown in Figure 2. Let the area for which one sensor is responsible be denoted by $A$. For the regular grid deployment $A=a^{2}$.

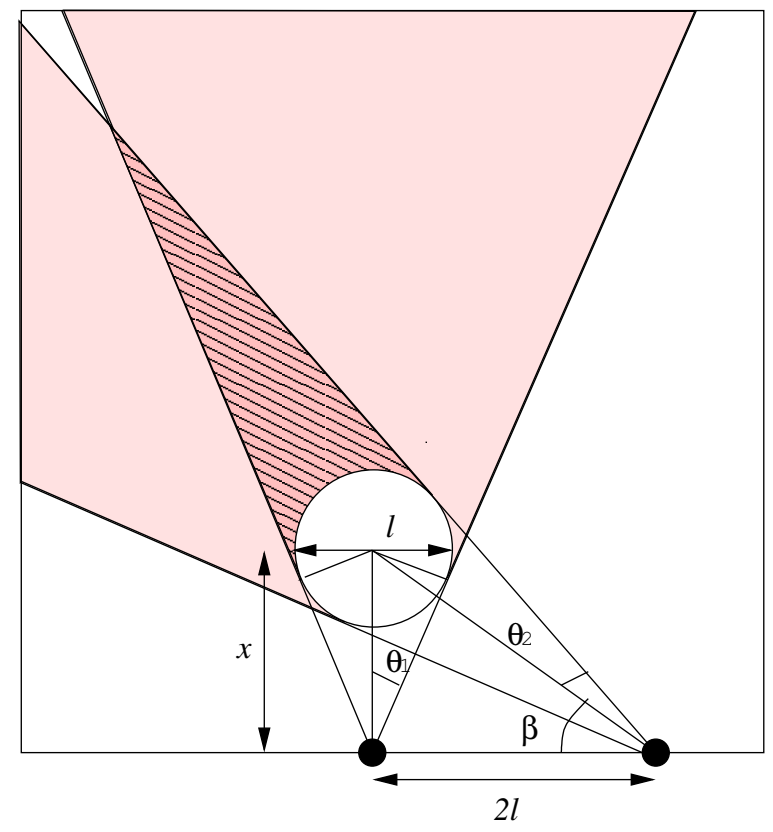

Figure 2: Abstract obstacle model for analytical calculation of coverage.
Consider a circular obstacle of diameter $l$ present in this tile which blocks the coverage of the sensor. We will assume that the sensor is capable of limited mobility over a range which is a small multiple of $l$.

Suppose the area occluded by the obstacle is $A_{\text {occluded }}$ (shown shaded in Figure 2). To quantify the coverage, we define the probability of mis-detection, $P o M$, as

$$
P o M=\frac{1}{A} \int_{A_{\text {occluded }}} f_{\text {target }}(x, y) d x d y
$$

where $f_{\text {target }}(.,$.$) is the probability density of the target location$ within $A$.

We assume no design time knowledge of the target location which leads to a uniform $f_{\text {tar get }}(.,$.$) . For this case, P o M=A_{\text {occluded }} / A$.

When no obstacle is present, $A$ is completely covered by the sensor and hence $P o M=0$.

Now consider the case when one obstacle is present. We analyze for one obstacle position to illustrate our point, but the analysis can be carried out for any location of the obstacle. Consider the position of the obstacle shown in Fig. 2 such that both the tangents from the sensor to the obstacle circle meet the top edge of the tile. Let $\theta_{1}$ be the angle made by the tangent to the obstacle edge with the vertical line joining the sensor and the center of the obstacle. Let the distance of the obstacle center from the sensor be $x$. Then,

$$
\begin{array}{r}
A=a^{2}-\pi\left(\frac{l}{2}\right)^{2} \\
A_{\text {occluded }}^{\text {static }}=a^{2} \tan \theta_{1}-\frac{l^{2}}{4 \tan \theta_{1}}-\left(\pi+\theta_{1}\right) \frac{l^{2}}{8}
\end{array}
$$

where $\theta=\sin ^{-1}(l / 2 x)$. Next consider the case where the sensor is capable of moving a small multiple of $l$, say $2 l$, along a track along the edge of the tile. The occluded area when the camera may use any location along its track to cover the tile is shown hashed in the figure. This area is

$$
A_{\text {occluded }}^{\text {mobile }}=\frac{l^{2}}{4} \tan (\omega)-\frac{\omega l^{2}}{4}
$$

where

$$
\begin{array}{r}
\omega=\frac{\pi / 2+\theta_{1}+\theta_{2}+\beta}{2} \\
\theta_{2}=\sin ^{-1}\left(\frac{l / 2}{\sqrt{4 l^{2}+x^{2}}}\right) \\
\beta=\tan ^{-1} \frac{x}{2 l}
\end{array}
$$

To determine the magnitude of improvement for specific values of the model parameters, let us evaluate the gain due to mobility as the factor, $G$, by which the occluded area is reduced due to mobility.

$$
G=\frac{A_{\text {occluded }}^{\text {static }}}{A_{\text {occluded }}^{\text {mobile }}}
$$

using the expressions derived in equations 3 and 4 .

We evaluate the above equation for a sample set of parameter values. Fig 3 shows $G$ for $a=100, l \in(5,20)$ and the distance of obstacle, $x \in(20,35)$. These values are such that the geometrical calculations above hold; the relative values of $x, l$ and $a$ are such that the figure drawn above represents the situation correctly, the calculations will change if the shapes of occluded areas differ. 


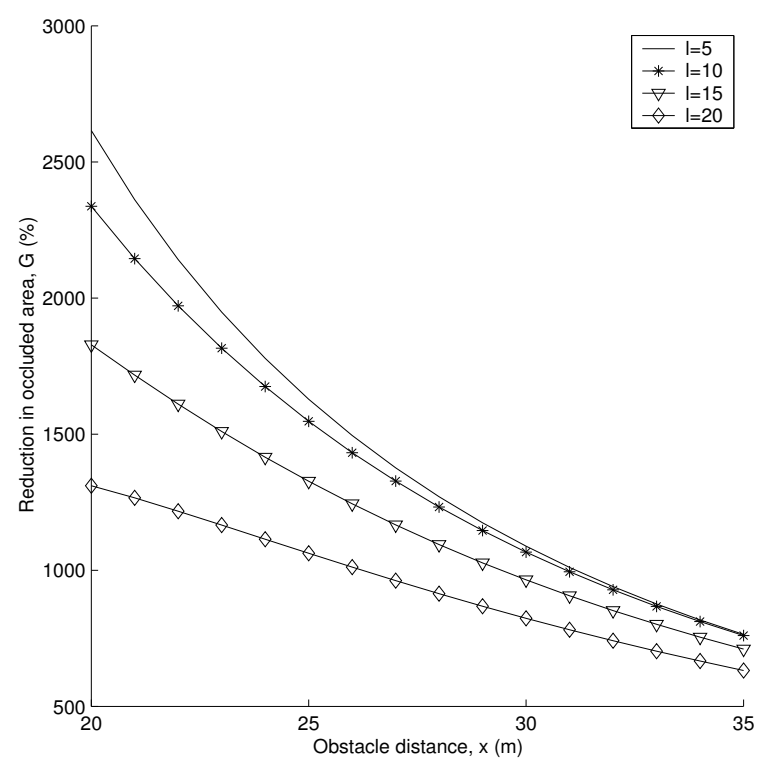

Figure 3: Calculating the advantage due to mobility for a simplified obstacle model.

Figure 3 shows clearly that limited range mobility reduces the occluded area by up to an order of magnitude in the above cases. This mobility offers a dramatic impact, therefore, on detection probability for distributed sensors with only a small resource cost addition due to adding this mobility capability.

Analytical calculation may be continued for varying positions of the obstacle and may be extended for more than one obstacle. However, analysis becomes intractable as the number of obstacles grows and we resort to simulations for evaluating more complex and representative environments.

\section{LOW COMPLEXITY ACTUATION IN SEVERAL ENVIRONMENTS}

We now consider several scenarios with multiple obstacles. The simulations essentially model a surface deployment, where the sensors are mounted on objects lying on the ground, pillars or trees. The effect of height is not accounted for in the obstacle model. Three dimensional calculations would be needed when sensors are observing the environment from a UAV or very high altitude.

Again, we consider sensors placed along edges of a square region, which as before models one tile of a large deployment. The mobile sensor is assumed to be able to move a short distance along the edge.

To model realistic obstacles, we first note than most everyday objects have a small aspect ratio. Also, for the line of sight sensor, it is not the exact shape of the obstacle but the angle subtended by it at the sensor which determines the occlusion. With this reasoning, we simulate the obstacles as circular. A notable exception to small aspect ratio objects are walls and other forms of boundaries which will severely limit the coverage of a sensor and we do not expect low complexity actuation to overcome the effect of walls.

The obstacle diameter is assumed to be a random variable with uniform distribution, between 0 and $2 l_{a v}$. The density of obstacles is represented as number of obstacles per unit area. The obstacles are placed uniformly randomly over a square area of size $100 \times 100$. The random coordinates may lead to overlapping obstacles causing the formation of complex obstacle shapes. As discussed before, we are not concerned with the exact shape of obstacle but rather with the occlusion caused by it. The sensor is again assumed to be capable of moving a distance $d_{\text {move }}$ which is a small multiple of $l_{a v}$.

Coverage is measured by evaluating the area which is visible to the sensor compared to the free area left in the square after the area occupied by the obstacles themselves is subtracted. The line of sight from the sensor to every point in the free area is tested and if there is an obstacle blocking it that point is assumed occluded. Coverage can be calculated by counting the occluded points and the visible points.

To suppress the effect of peculiar chance placements, for each choice of parameter values we average our measurements over 20 runs of the simulation. One of the sample obstacle placements is shown in Figure 4, with one sensor placed along the lower edge.

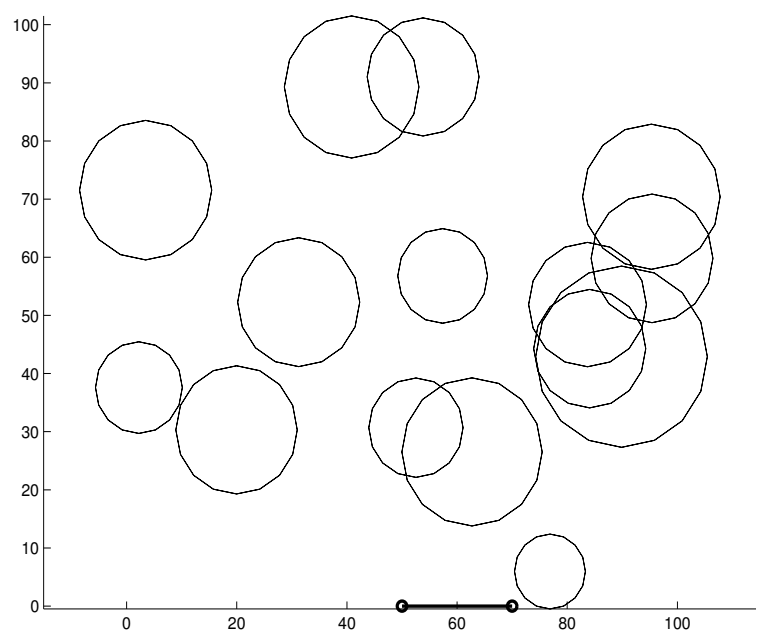

Figure 4: Sample obstacles in deployment terrain (The small line along the lower edge shows the track on which the sensor moves).

For the first simulation, there is one sensor placed at the center of the lower edge. The value of $l_{a v}$ is $5 \mathrm{~m}$. The obstacle density is varied from 1 obstacles in the square region to 15 obstacles. Coverage is evaluated for three cases: when there is no mobility, when the sensor can move $l_{a v}$ to one side of the center position and when the sensor can move $2 l_{a v}$, equally divided on both sides of the center position. Figure 5 shows the fraction of free area covered in each case. For each obstacle density, coverage is obtained by averaging over 20 obstacle placements, in each of the three sensor mobility cases.

The gains due to mobility in varying obstacle density, compared to a static sensor are shown in Figure 6. The gain is defined as:

$$
G=\frac{\text { PoM }_{\text {static }}}{\text { PoM mobile }}
$$

The figure shows $200 \%$ to $700 \%$ gain for sensors with small mobility compared to static sensors. Considering the fact that sensing is the key functionality of a sensor network, these gains are very significant.

The next simulation studies the advantage due to mobility in varying obstacle size. The value of $l_{a v}$ varies between 5 and $20 \mathrm{~m}$ in a $100 \times 100$ area. The number of obstacles is kept fixed at 10 . Figure 7 shows the multiplicative reduction in mis-detection probability, G. The labels 1 and 2 along the $\mathrm{x}$-axis refer to cases when 


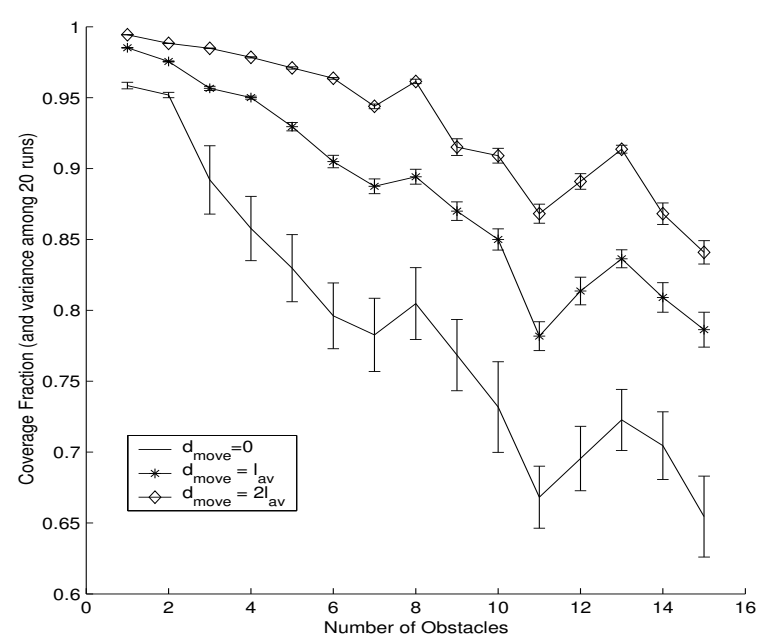

Figure 5: Coverage with varying obstacle density, with and without mobility. The error bars show the variance among 20 runs with different random obstacle placements.

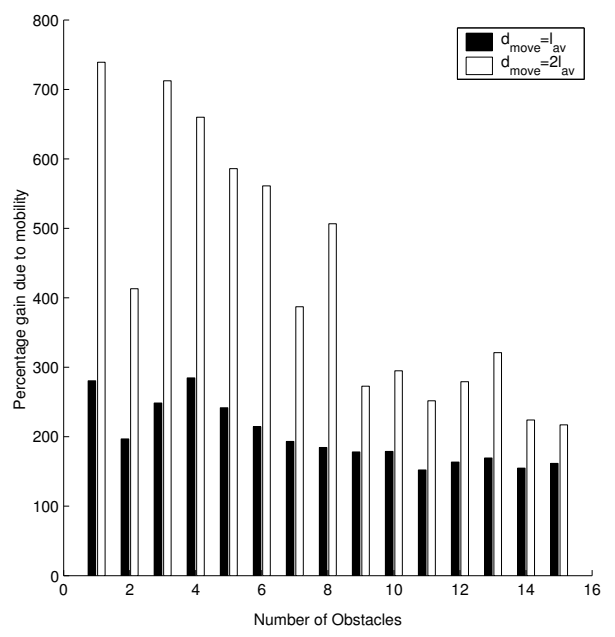

Figure 6: Actuation advantage (multiplicative reduction in probability of mis-detection) in varying obstacle density.

the sensor is allowed to move $l-a v$ and $2 l_{a v}$ respectively. Again, actuation shows significant advantage, giving more than a factor of 2 improvement. The variance in the results from the 20 random topologies, not plotted for brevity, is less than 0.05 in all cases.

The simulation results are very encouraging and verify that the performance gains expected in simplified analysis with a single obstacle are also expected with realistic scenarios having multiple obstacles and varying camera mobility range. These results motivate us to implement experimental systems which utilize actuation capability.

\section{EXPERIMENTAL RESULTS}

We extended our simulations with a real sensor detecting a target in the presence of obstacles. This section describes the camera testbed built for this purpose and our experiments on it in two different scenarios - a controlled laboratory environment and an outdoor environment with trees and foliage.

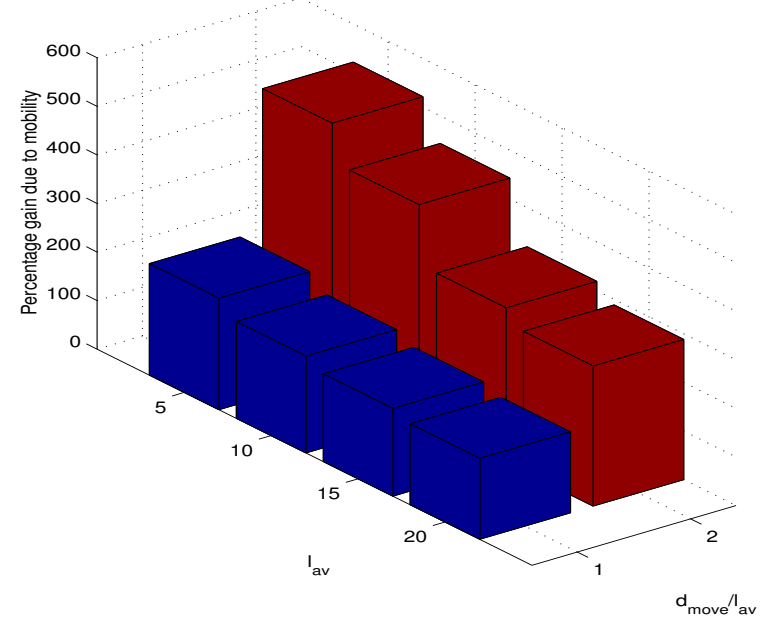

Figure 7: Actuation advantage (multiplicative reduction in probability of mis-detection) with varying obstacle size.

\subsection{Laboratory Test-bed with Cameras and Obstacles}

Figure 8 shows a picture of obstacles in our laboratory test-bed, seen from the camera location.

The sensor used on the test-bed is an Axis 2100 camera system. [38]. The camera system is equipped with rotational articulation to enable imaging in the entire plane of rotation.

Of course, these imager systems are line of sight sensors, but practical considerations which have not been included in the analysis apply here. For instance, in actual field deployment, the sensor angle of view may be constrained and illumination conditions may affect detection. The effects of these phenomena were reduced here by providing adequate lighting. Object detection was assured at the maximum range in this experiment for conditions where line of sight observation was allowed by obstacles.

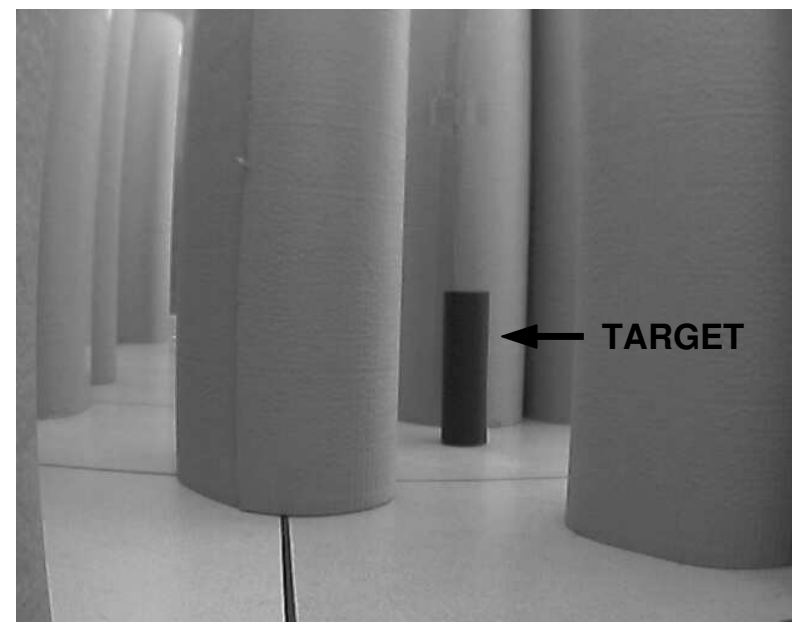

Figure 8: The laboratory test-bed for testing coverage advantage due to mobility.

The coordinates for obstacle placement are obtained from the actual location of trees in the Wind River forest [39]. The size of tree stems is assumed equal for simplicity of construction. Actual tree coordinates do not follow a uniformly random distribution due to 
physiological phenomena and using actual forest tree coordinates is expected to provide a realistic obstacle scenario. The obstacles used here are cylinders with diameter of 1 foot. They are placed in a 12 foot by 12 foot grid. The tree coordinates are shown in Figure 9.

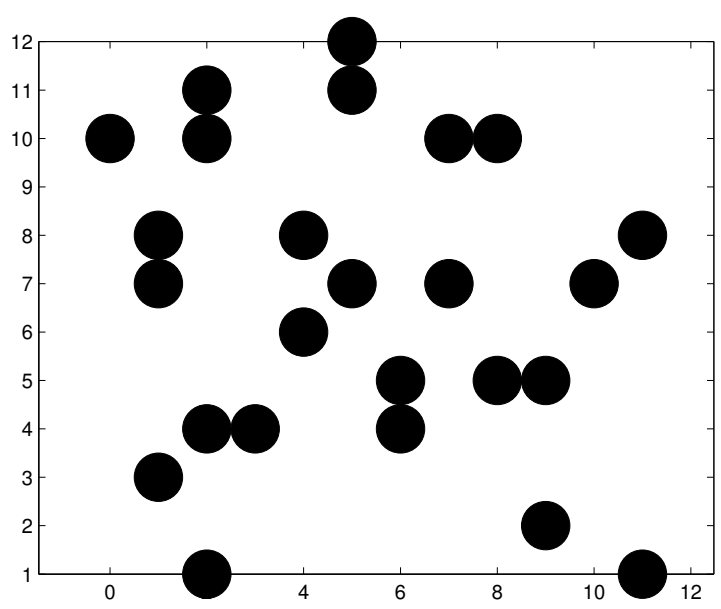

Figure 9: The coordinates of trees from Wind River forest [39] used to place the obstacles in laboratory test-bed.

The target itself is a small cylinder of a different color than the obstacles. The obstacles are colored brown to abstract tree stems. A simple image processing technique is used to detect the target in the captured image. As the lighting in the laboratory is controlled, we can take an image of the background with no target present and detect the target by subtracting an image taken when the target is present from the background image. If the target is completely occluded, it is assumed not detected. If it is partially occluded, we assume it as detected if the number of pixels visible is above a certain threshold. This threshold procedure reduces the contribution of noise associated with the camera system by ensuring that a minimum portion of the target is observed in order to declare a positive detection.

The experiment is performed as follows. First the camera is placed at the midpoint of one edge of the square area. With the camera stationary, the target is moved to uniformly spaced locations on the $12^{\prime} \times 12^{\prime}$ grid. The number of locations at which the camera is able to detect the target divided by the total number of locations at which the target was placed gives the coverage achieved by the static sensor.

Next the camera is assumed to be able to move a distance of two feet. Coverage is again computed using the previous target placement procedure but now if the target can be detected by the camera by moving along its track, the target is assumed detected.

Further, we vary the number of cameras available. One additional camera is successively added on each of the other edges. The mis-detection probabilities are plotted in Figure 10 for varying number of cameras, both for the stationary case and the mobile case. The mis-detection with mobility reduces significantly compared to the static case, reaching an order of magnitude improvement in some cases.

\subsection{Experiment in Trees and Foliage}

Previous work on coverage in sensor networks $[15,16,17]$ has been based only on abstract simulations and not on measurements

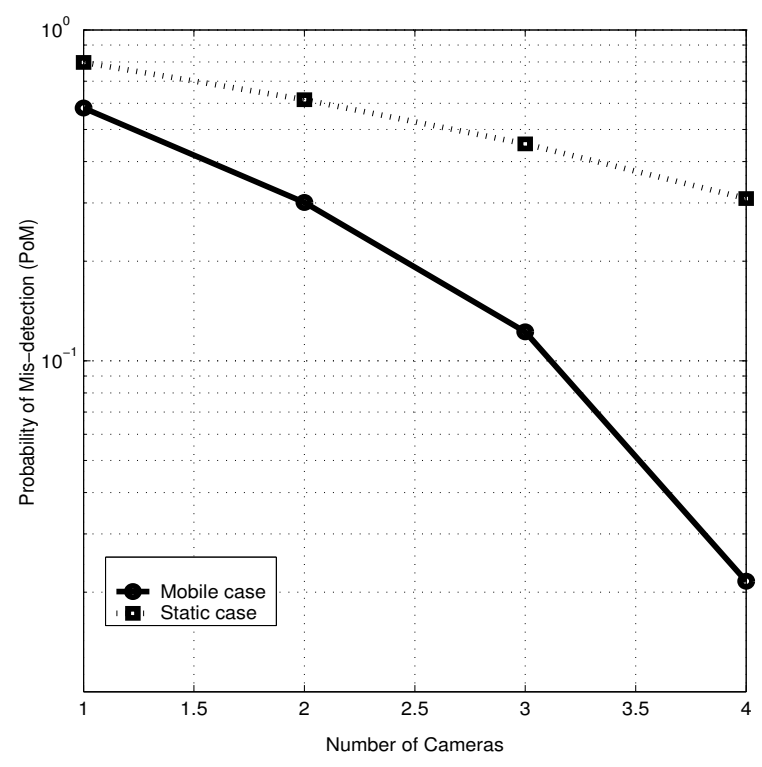

Figure 10: Probability of Mis-detection in laboratory test-bed experiments: varying number of cameras. PoM is lower with mobility.

with real sensors; our experiment is one of the first attempts to corroborate the proposed coverage enhancement methods with realworld measurements.

We test our findings in an environment with trees and foliage occluding the scene. The location chosen was a forested area near our campus, Figure 11.

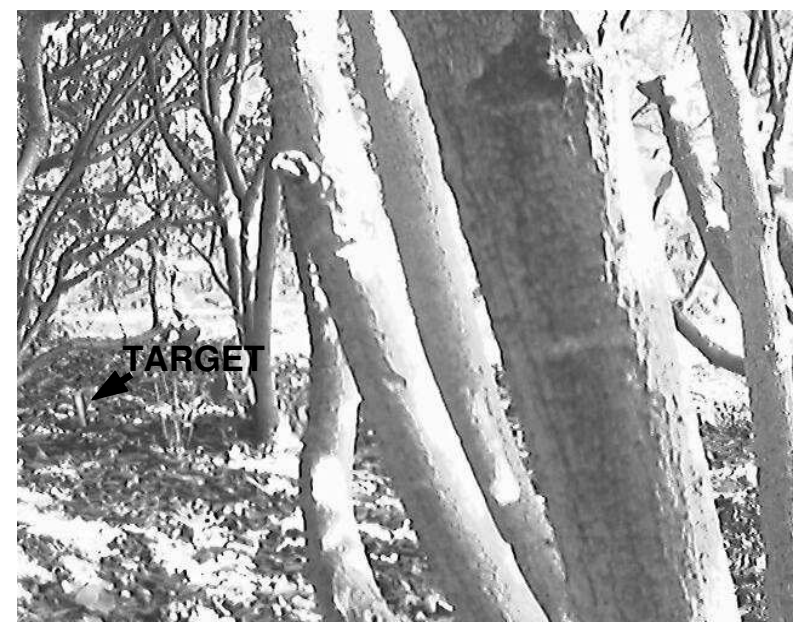

Figure 11: Image of real world scene showing obstacles consisting of trees and foliage, among which the target is to be detected.

The obstacles are no longer ideal cylinders and cameras do not operate in controlled lighting conditions. The cameras used as sensors are not designed for outdoor usage and the image quality is affected by exposure to sunshine for the long duration required for collecting data in our experiments. This leads to some errors in our simple image processing techniques for detecting the target. The experimental challenges include operating from batteries away from wall sockets and positioning the sensors on uneven terrain 
instead of the levelled laboratory floor. It may be noted that the foliage causes rather large occlusions and our mobility here is much less than the mean obstacle diameter, instead of being an integral multiple of it.

The experiment performed consists of one camera placed along one edge. Coverage is measured over a $12^{\prime} \times 12^{\prime}$ grid in the forest. Detection probability is first measured when the camera is fixed at the midpoint of the edge. Then the camera is allowed to move two feet in one direction. Third the camera is allowed to move 2 feet in both directions. The mis-detection probabilities and gains are tabulated in Table 1 . The measurements show that mobility even when lower than average obstacle length is able to provide significant advantage in reducing the uncertainty of sensing.

Table 1: Mis-detection probabilities with and without motion for real-world experiment

\begin{tabular}{|c|c|c|}
\hline CameraMode & PoM & G (\%)(relative to static case) \\
\hline STATIC & 0.3885 & - \\
\hline MOVE, 1 direction & 0.2374 & 163.65 \\
\hline MOVE, 2 directions & 0.1942 & 200.05 \\
\hline
\end{tabular}

\section{ASSESSMENT}

The intuition suggested by the analytical results regarding the benefits of limited actuation is extensively tested through simulations, lab experiments and real world tests. Our study establishes a clear advantage gained by introducing a very limited low complexity and low energy motion capability.

These findings are very important for practical system design in several situations. The cost of sensors, such as high resolution imagers, may be too high to deploy in the large numbers required for complete coverage. Motion, without its dependencies of navigation, localization and terrain feedback reduces to simply providing traction mechanisms such as a motor and possibly a track to reduce energy consumption when moving. This kind of mobility is cheap to provide and easy to control. Thus in situations where the latency of motion is not a severe constraint, our method provides the designer with a valuable trade-off to optimize the system design and performance. The low complexity actuation primitives introduced are also useful for adapting to the changing environment dynamics such as moving obstacles and introduction of new occlusions.

This work is now being extended in two directions. One is building the obstacle detection modules which can be added to sensor nodes to learn the presence of obstacles in the scene. This information can then be used by the sensor node to decide its position or to plan its motion. The second is the development of collaborative algorithms for sensor nodes to optimize their positions in view of the learnt propagation characteristics of the environment. These methods can enable a set of randomly placed nodes to customize their positions for the specific deployment and update it as required.

\section{CONCLUSIONS}

We have suggested a new method to improve sensing performance under the specific feasibility constraints of sensor networks. Our method allows the system to work in realistic deployment scenarios with obstacles and changing occlusions. Apart from analytical and simulation verification, we tested our proposal on real sensors deployed in a physical environment with abundant trees and foliage acting as obstacles. This is the first attempt at developing methods which work with realistic environmental models as opposed to previous methods which make several idealistic assumptions.

Future work includes exploring the exact trade-offs between the number of mobile and static sensors in relation to various performance and cost constraints. We also intend to study the advantages of limited mobility for communication and other services in the sensor network.

\section{ACKNOWLEDGMENTS}

The authors wish to acknowledge the contributions of Eric Lin, Michael Smith, and Michael Stealey. Gratitude is also extended to the Center for Embedded and Networked Sensing (CENS), at University of California, Los Angeles.

This material is based upon work supported by the National Science Foundation (NSF) under Grant Nos. ANI-00331481 and ECS0225379. Any opinions, findings and conclusions or recommendations expressed in this material are those of the author(s) and do not necessarily reflect the views of the NSF.

\section{REFERENCES}

[1] A Savvides and Mani B Srivastava. A distributed computation platform for wireless embedded sensing. In IEEE nternational Conference on Computer Design: VLSI in Computers and Processors, pages 220 -225, September 2002.

[2] G. J. Pottie and W. J. Kaiser. Wireless integrated network sensors. Communications of the ACM, 43(5):51-58, 2000.

[3] Deborah Estrin, Ramesh Govindan, and John Heidemann. Embedding the internet: introduction. Communications of the ACM, 43(5):38-41, 2000.

[4] J Rabaey, J Ammer, J.L da Silva Jr., and D Patel. Picoradio: Ad-hoc wireless networking of ubiquitous low-energy sensor/monitor nodes. pages 9-12, Orlando, Fl, USA, April 2000.

[5] Tinyos: a component based os for the networked sensor regime. http://webs.cs.berkeley.edu/tos/.

[6] P Steurer and MB Srivastava. System design of smart table. In Proceedings of the First IEEE International Conference on Pervasive Computing and Communications (PerCom 2003), pages 473 -480, March 2003.

[7] M Srivastava, R. Muntz, and M. Potkonjak. Smart kindergarten: sensor-based wireless networks for smart developmental problem-solving enviroments. In Proceedings of the Seventh ACM Annual International Conference on Mobile Computing and Networking (MobiCom), pages 132 138, July 2001.

[8] Multimodal networks of in-situ sensors. http://mantis.cs.colorado.edu/.

[9] Alberto Cerpa, Jeremy Elson, Deborah Estrin, Lewis Girod, Michael Hamilton, and Jerry Zhao. Habitat monitoring: Application driver for wireless communications technology. In ACM SIGCOMM Workshop on Data Communications in Latin America and the Caribbean, 2001.

[10] Alan Mainwaring, Joseph Polastre, Robert Szewczyk, David Culler, and John Anderson. Wireless sensor networks for habitat monitoring. In First ACM Workshop on Wireless Sensor Networks and Applications, Atlanta, GA, USA, September 2002.

[11] Jeff Burke, Eitan Mendelowitz, Joseph Kim, and Rex Lorenzo. Networking with knobs and knats: Towards 
ubiquitous computing for artists. In Ubiquitous Computing, Concepts and Models Workshop, Gothenburg, Sweden, 2002.

[12] Mohammed Rahimi, Hardik Shah, Gaurav S. Sukhatme, John Heidemann, and D. Estrin. Studying the feasibility of energy harvesting in a mobile sensor network. In IEEE Int'l Conference on Robotics and Automation, 2003.

[13] Rahul C Shah, Sumit Roy, Sushant Jain, and Waylon Brunette. Datamules: Modelling a three tiered architecture for sparse sensor networks. In First IEEE International Workshop on Sensor Network Protocols and Applications (SNPA), May 2003.

[14] Gabriel T. Sibley, Mohammad H. Rahimi, and Gaurav S. Sukhatme. Robomote: A tiny mobile robot platform for large-scale ad-hoc sensor networks. In IEEE International Conference on Robotics and Automation, Washington, DC, USA, September 2002.

[15] Xiaorui Wang, Guoliang Xing, Yuanfang Zhang, Chenyang $\mathrm{Lu}$, Robert Pless, and Christopher Gill. Integrated coverage and connectivity configuration in wireless sensor networks. In ACM SenSys 2003, Los Angeles, USA, November 2003.

[16] Seapahn Meguerdichian, Farinaz Koushanfar, Miodrag Potkonjak 1, and Mani B. Srivastava. Coverage problems in wireless ad-hoc sensor networks. In Twentieth Annual Joint Conference of the IEEE Computer and Communications Societies (INFOCOM), volume 3, pages 1380-1387, April 2001.

[17] Xiang-Yang Li, Peng-Jun Wan, and Ophir Frieder. Coverage in wireless ad hoc sensor networks. IEEE Transactions on Computers, 52(6), June 2003.

[18] Honghia Zhang and Jennifer C. Hou. Maintaining sensing coverage and connectivity in large sensor networks. Technical Report UIUCDCS-R-2003-2351, University of Illinois, Urbana Champagne, June 2003.

[19] Chi-Fu Huang and Yu-Chee Tseng. The coverage problem in a wireless sensor network. In Proceedings of the 2nd ACM international conference on Wireless sensor networks and applications, pages 115-121. ACM Press, 2003.

[20] Bulent Yener, M. Inanc, and M. Magdon-Ismail. Power optimal connectivity and coverage in wireless sensor networks. Technical Report TR-03-06, Department of Computer Science, Rensselaer Polytechnic Institute, July 2003.

[21] Abiola Fanimokun and Jeff Frolik. Effects of natural propagation environments on wireless sensor network coverage area. In Southeastern Symposium on System Theory (SSST), March 2003.

[22] T.S. Rappaport, K. Blankenship, and H. Xu. Propagation and radio system design issues in mobile radio systems for the glomo project.

http://www.mprg.ee.vt.edu/research/glomo/prev2.html, January 1997.

[23] Edward H. Adelson and James R. Bergen. Computational Models of Visual Processing, chapter The Plenoptic Function and the Elements of Early Vision, pages 3-20. MIT Press, 1991.

[24] Jin-Xiang Chai, Shing-Chow Chan, Heung-Yeung Shum, and Xin Tong. Plenoptic sampling. In Proceedings of the 27 th annual conference on Computer graphics and interactive techniques, pages 307-318. ACM Press/Addison-Wesley Publishing Co., 2000.

[25] William H. Bares, Somying Thainimit, and Scott McDermott. A model for constraint-based camera planning.
In AAAI Spring Symposium Series Smart Graphics, March 2000.

[26] J. Maver and R. Bajcsy. Occlusions as a guide for planning the next view. IEEE Transactions on Pattern Analysis and Machine Intelligence, 15(5):417-433, May 1993.

[27] V. A. Sujan. Task directed imaging in unstructured environments by cooperating robots. In The Third Indian Conference on Computer Vision, Graphivs and Image Processing, Ahmedabad, India, December 2002.

[28] H.H. Gonzalez-Banos, C.Y. Lee, and J.C. Latombe. Real-time combinatorial tracking of a target moving unpredictably among obstacles. In IEEE International Conference on Robotics and Automation, Washington DC, USA, May 2002.

[29] B Jung and GS Sukhatme. Tracking targets using multiple robots: The effect of environment occlusion. Autonomous Robots Journal, 13(3):191-205, 2002.

[30] Matthias Grossglauser and David N. C. Tse. Mobility increases the capacity of ad hoc wireless networks. IEEE/ACM Transactions on Networking (TON), 10(4):477-486, 2002.

[31] Srdjan Capkun, Jean-Pierre Hubaux, and Levente Buttyn. Mobility helps security in ad hoc networks. In Proceedings of the 4th ACM international symposium on Mobile ad hoc networking and computing, pages 46-56. ACM Press, 2003.

[32] Philo Juang, Hidekazu Oki, Yong Wang, Margaret Martonosi, Li Shiuan Peh, and Daniel Rubenstein. Energy-efficient computing for wildlife tracking: design tradeoffs and early experiences with zebranet. In Tenth international conference on architectural support for programming languages and operating systems (ASPLOS-X), pages 96-107. ACM Press, 2002.

[33] Tara Small and Zygmunt J. Haas. The shared wireless infostation model: a new ad hoc networking paradigm (or where there is a whale, there is a way). In Proceedings of the 4th ACM international symposium on Mobile ad hoc networking and computing, pages 233-244. ACM Press, 2003.

[34] A Chakrabarty, A Sabharwal, and B Aazhang. Using predictable observer mobility for power efficient design of a sensor network. In Second International Workshop on Information Processing in Sensor Networks(IPSN), pages 129-145, Palo Alto, CA, USA, April 2003.

[35] G. Kesidis, T. Konstantopoulos, and S. Phoha. Surveillance coverage of sensor networks under a random mobility strategy. In IEEE Sensors, October 2003.

[36] A Howard, MJ Mataric, and GS Sukhatme. An incremental self-deployment algorithm for mobile sensor networks. Autonomous Robots Journal (Special Issue on Intelligent Embedded Systems), 13(2):113-126, 2002.

[37] W. Merrill, L. Girod, J. Elson, K. Sohrabi, F. Newberg, and W. Kaiser. Autonomous position location in distributed, embedded, wireless systems. In IEEE CAS Workshop on Wireless Communications and Networking, Pasadena, CA, September.

[38] Axis 2100 network camera. Product Datasheet, Axis Communications, http://www.axis.com/documentation/datasheet/2100/2100 _ds.pdf.

[39] Wind river canopy crane research facility. http://depts.washington.edu/wrccrf/12haplot/. 12-ha Permanent Plot Tree Data. 